JOURNAL OF SYNCHROTRON RADIATION

ISSN 1600-5775

Received 19 September 2017

Accepted 19 December 2017

Edited by S. M. Heald, Argonne National Laboratory, USA

Keywords: RIXS; high resolution; analyzer; inelastic X-ray; quartz.

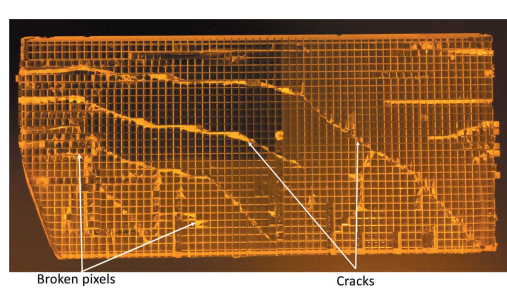
OPEN $\odot$ ACCESS

\section{High-energy-resolution diced spherical quartz analyzers for resonant inelastic $\mathrm{X}$-ray scattering}

\author{
Ayman H. Said, ${ }^{\mathrm{a} *}$ Thomas Gog, ${ }^{\mathrm{a}}$ Michael Wieczorek, ${ }^{\mathrm{a}}$ XianRong Huang, \\ Diego Casa, ${ }^{a}$ Elina Kasman, ${ }^{a}$ Ralu Divan ${ }^{b}$ and Jung Ho Kim ${ }^{a}$ \\ ${ }^{\mathbf{a}}$ Advanced Photon Source, Argonne National Laboratory, USA, and ${ }^{\mathbf{b}}$ Center for Nanoscale Materials, \\ Argonne National Laboratory, USA. *Correspondence e-mail: said@aps.anl.gov
}

A novel diced spherical quartz analyzer for use in resonant inelastic X-ray scattering (RIXS) is introduced, achieving an unprecedented energy resolution of $10.53 \mathrm{meV}$ at the Ir $L_{3}$ absorption edge $(11.215 \mathrm{keV})$. In this work the fabrication process and the characterization of the analyzer are presented, and an example of a RIXS spectrum of magnetic excitations in a $\mathrm{Sr}_{3} \mathrm{Ir}_{2} \mathrm{O}_{7}$ sample is shown.

\section{Introduction}

Resonant inelastic X-ray scattering (RIXS) is a powerful spectroscopic technique for probing magnetic and charge excitations in a vast number of materials of interest for both basic and applied research (Ament et al., 2011). In recent years RIXS has gained remarkable popularity due to improvements in the energy resolution at more absorption edges of diverse elements.

Currently, the material of choice for RIXS analyzers is silicon $(\mathrm{Si})$, given the availability of very high quality single crystals in large boules and the maturity of fabrication techniques including cutting, polishing, bonding and etching (Sette et al., 1995; Masciovecchio et al., 1996; Schwoerer-Böehning et al., 1997).

The resonant character of the technique inevitably fixes the energy to the selected absorption edge and Si suffers from a limited selection of suitable reflections near backscattering for a given edge energy due to the high symmetry of its crystal lattice. Note that the Bragg angle has to be very close to backscattering (typically $<5^{\circ}$ ) in order to: (i) achieve the desired throughput (close to backscattering, the angular acceptance increases); and (ii) achieve energy resolution (the energy resolution is less sensitive to the angular divergence close to backscattering) (Kushnir \& Suvorov, 1990; Gog et al., 2013; Shvyd'ko, 2004). Therefore, there is an increased need to develop RIXS analyzers with other materials to improve energy resolution at various absorption edges overcoming the limitation of crystalline Si. Naturally, crystals with lower lattice symmetry such as sapphire $\left(\mathrm{Al}_{2} \mathrm{O}_{3}\right)$, quartz $\left(\mathrm{SiO}_{2}\right)$ and lithium niobate $\left(\mathrm{LiNbO}_{3}\right)$ would offer many more possible reflections to match diverse absorption edges, with various choices of intrinsic energy resolution and throughput depending on the experiment design (Gog et al., 2013).

RIXS analyzers working at relatively hard X-ray energies $(7-12 \mathrm{keV})$ require high-quality crystals that are uniform and defect-free over large areas. To date this requirement has proven too stringent for available sapphire and lithium niobate crystals. On the other hand, high-quality large single 
quartz crystal boules are commercially available, as shown in recent studies (Hönnicke et al., 2013; Sutter et al., 2005, 2006). In addition to crystal quality, quartz offers a multitude of reflections with narrower intrinsic width than silicon or sapphire (Fig. 1) making quartz very suitable for high-energy resolution analyzers.

There are many technical challenges associated with fabricating quartz analyzers including dicing, bonding and strain relief. Much effort has been invested in overcoming these challenges. In recent years, there have been new developments in fabricating sapphire and quartz analyzers for RIXS (Yavaş et al., 2007; Ketenoglu et al., 2015; Honnicke et al., 2016), and the best energy resolution achieved was $25 \mathrm{meV}$ for a quartz analyzer (Ketenoglu et al., 2015) at the $\mathrm{Cu} K$ absorption edge.

Other issues with quartz are its very low thermal conductivity and rather large linear thermal expansion. The thermal conductivity of quartz is $\sim 15$ times lower than that of silicon, which poses a difficulty when temperature is used to scan the analyzer energy. Similarly, the linear thermal expansion is significantly larger than that of silicon, and also anisotropic (Kosinski et al., 1991). Such high thermal expansion coefficient values require a very high thermal stability to maintain optimal energy resolution: a small thermal gradient over the analyzer surface or an overall temperature change over the course of a data scan would smear the resulting energy spectrum.

In this work, the achievement of an overall energy resolution of $10.53 \mathrm{meV}$ at the $\operatorname{Ir} L_{3}$ absorption edge $(E=$ $11.215 \mathrm{keV}$ ) is reported, using the RIXS beamline 27-ID at the Advanced Photon Source (APS), USA. An incident beam with a narrow bandpass of $8.9 \mathrm{meV}$ is generated by a high-

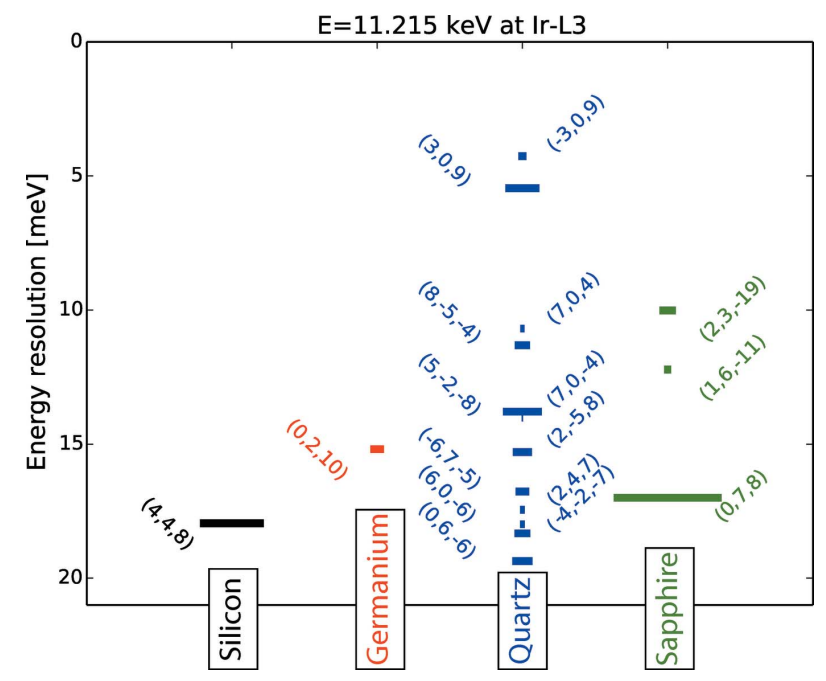

Figure 1

Analyzer energy resolution for different crystal analyzer materials at the Ir $L_{3}$ absorption edge $(E=11.215 \mathrm{keV})$; the length of the dash marker for each reflection represents the relative integrated reflectivity. The analyzer energy resolution shown in the figure is approximated as $\Delta E_{\mathrm{a}}=$ $\left[\Delta E_{\mathrm{i}}^{2}+\Delta E_{\mathrm{g}}^{2}\right]^{1 / 2}$, where $\Delta E_{\mathrm{i}}$ and $\Delta E_{\mathrm{g}}$ are the intrinsic and detector geometrical contributions, respectively, the latter calculated for a $2 \mathrm{~m}$ diameter Rowland circle and a strip detector pitch of $50 \mu \mathrm{m}$. These contributions are described in more detail in earlier work (Gog et al., 2013). heat-load diamond (111) and four-bounce symmetric Si (844) high-resolution monochromator, focused by a KB mirror to a size of approximately $10 \mu \mathrm{m} \times 40 \mu \mathrm{m}(\mathrm{V} \times \mathrm{H})$ full width at half-maximum (FWHM) at the sample position. Scattered radiation from the sample is collected by a diced spherical quartz analyzer and refocused onto a $50 \mu \mathrm{m}$-pitch positionsensitive (strip-) detector on a 2 m-diameter Rowland circle. For the analyzer, the quartz (309) reflection was selected, which has an intrinsic bandpass of $3.7 \mathrm{meV}$ at the $\operatorname{Ir} L_{3}$ edge.

\section{Fabrication}

The steps in the fabrication of the quartz analyzer presented in this work are described later schematically in Fig. 4 and detailed below.

\subsection{Preparation of quartz wafer}

Quartz wafers (2 mm thick, $25 \mathrm{~mm}$ diameter) were sliced from a high-quality $\alpha$-quartz ingot, grade $\mathrm{A}$, inclusion Ia, which was purchased from Tokyo Denpa Co. Ltd in Japan. The flat surfaces were fine ground and then etched in hydrofluoric acid (HF) standard solution (48\% $\mathrm{HF}$ and $52 \%$ water by volume) at room temperature for $8-10 \mathrm{~h}$ to remove about 80 $100 \mu \mathrm{m}$ from each side of the wafer; this is necessary to remove the residual strain from cutting and fine grinding (Fig. 4, step 1). The wafers were then polished on both sides to optical grade using chemical-mechanical polishing to achieve strain-free surfaces (Fig. 4, step 2).

\subsection{Initial dicing step}

Dicing quartz wafers with non-standard orientations was found to be more challenging than anticipated and required additional effort to optimize the process. A significant problem encountered during dicing is cracking of the wafer and fragmentation of pixels as shown in Fig. 2(a). The problem was mitigated by reducing the wafer thickness to $\sim 2 \mathrm{~mm}$ and optimizing the dicing parameters as shown in Table 1. The wafer cracking and pixel fragmentation problem seem to persist for thicker ( $>2 \mathrm{~mm}$ ) quartz wafers. Quartz is a piezoelectric crystal and exhibits many interesting characteristics including triboluminescence which was indeed observed during dicing.

While quartz does not have clearly defined cleavage planes, a number of studies (Bloss \& Gibbs, 1963) have noted that, when crushed, quartz cracks more readily along the $r=$ $(1,0,-1,1)$ and $z=(0,1,-1,1)$ planes. While impossible to completely avoid cleavage planes, we have found experimentally that cracking is reduced when dicing parallel and normal to the $c$-axis projection on the wafer, as shown in Fig. 2(b).

For the first dicing step, the quartz was mounted on a support silicon wafer using low-melting-point holding wax (75175 holding wax by Universal Photonics Incorporated). The $2 \mathrm{~mm}$-thick quartz wafer was diced $1.75 \mathrm{~mm}$ deep, leaving a back-wall of $250 \mu \mathrm{m}$. Multiple $0.25 \mathrm{~mm}$-deep passes per groove were used (Fig. 4, step 3). 
(a)

(b)

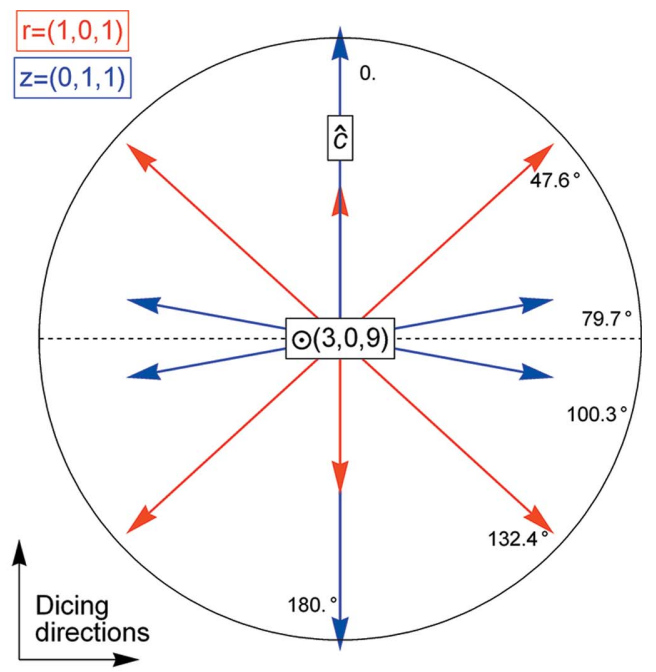

Figure 2

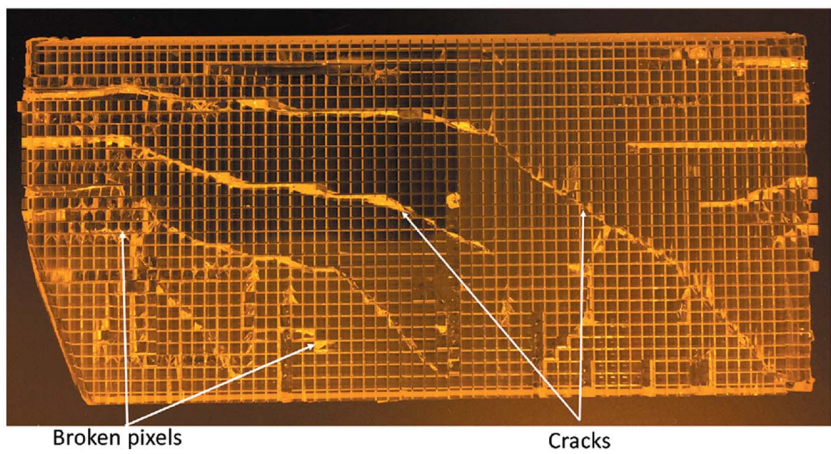

(a) Picture of a partially diced quartz wafer, about half way through the total thickness, showing cracks and areas left by pixel fragmentation.

(b) Schematic showing the direction of the diced grooves with respect to the projection of the $c$-axis and the $r$ and $z$ planes.

Table 1

Parameters used to dice quartz wafers.

\begin{tabular}{llllll}
\hline Blade type & $\begin{array}{l}\text { Blade } \\
\text { diameter } \\
(\mathrm{mm})\end{array}$ & $\begin{array}{l}\text { Cutting } \\
\text { speed } \\
\left(\mathrm{mm} \mathrm{s}^{-1}\right)\end{array}$ & $\begin{array}{l}\text { Spindle } \\
\text { speed } \\
(\text { r.p.m. })\end{array}$ & $\begin{array}{l}\text { Blade } \\
\text { thickness } \\
(\mu \mathrm{m})\end{array}$ & $\begin{array}{l}\text { Cut depth } \\
\text { per pass } \\
(\mu \mathrm{m})\end{array}$ \\
\hline $\begin{array}{l}\text { ADT resin-bond } \\
\text { blade }\end{array}$ & 100 & 0.2 & 12000 & 250 & 250 \\
\begin{tabular}{l} 
Grit size $=53 \mu \mathrm{m}$ \\
\hline
\end{tabular} & & & & \\
\hline
\end{tabular}

\subsection{First etch}

The quartz wafer was etched in HF standard solution at room temperature while still mounted on the silicon support wafer (Fig. 4, step 4). HF etches quartz without etching the silicon supporting wafer; the supporting wafer is needed as the diced quartz wafer is too fragile to handle. Alternatively, HFresistant plastic wafers could be used as supporting wafers. Etching quartz with HF is an anisotropic process; the surface of each pixel was etched faster than its sides as shown in Fig. 3. While the wax used to hold the quartz wafer to the silicon wafer is HF resistant, some HF did seep under the surface of the quartz wafer around its perimeter and slightly etched some parts of the lower surface.

\subsection{Gluing}

In order to dice the quartz wafer all the way through, the diced side was glued to a supporting silicon wafer, $0.5 \mathrm{~mm}$ thick. An HF-resistant two-component epoxy resin glue (Master Bond EP21ARHT) was spread over this new supporting wafer. The diced surface of the quartz wafer was then positioned over it with a small weight $(\sim 1 \mathrm{~kg})$ placed on top for a more consistent glue layer thickness. Once the glue was cured, the stack of wafers was heated to melt the holding wax in order to remove one of the support wafers. Then the diced quartz surface was cleaned of wax residue (Fig. 4, step 5).

\subsection{Final dicing step}

The quartz wafer was diced from the other side after aligning the blade to the grooves on the first side. The final dicing was performed using a $250 \mu \mathrm{m}$ blade through the remaining thickness, thus leaving an array of quartz pixels glued onto the silicon wafer (Fig. 4, step 6).

\subsection{Final etch}

The last dicing step left residual strain near the top $250 \mu \mathrm{m}$ of the quartz pixels; consequently, the diced quartz wafer needed to be etched again. The whole assembly (diced pixels mounted on the silicon wafer) was placed in standard HF solution for $3 \mathrm{~h}$ intermittently at room temperature, $1 \mathrm{~h}$ at a time while rinsing with water in between to reduce degrada-

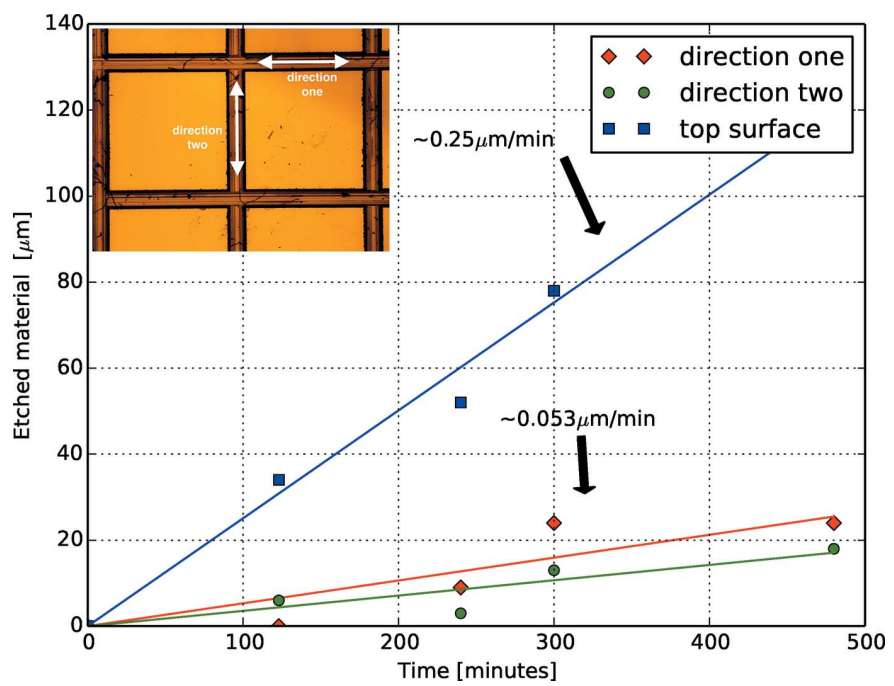

Figure 3

Etching rate of quartz from the top surface as well as from the sides. The side-etching rate quoted here is from both sides of the groove, half of that would correspond to the etching rate from one side of a step. The inset shows an image of a small part of the diced wafer before etching. 
tion of the glue (Fig. 4, step 7). While the glue was HF resistant, HF managed to seep between the surface of the silicon wafer and the glue layer which resulted in some delamination. Alternatively, only the top part of the quartz pixels could be submerged in HF while holding the assembly upside down.

\subsection{Bending to $2 \mathrm{~m}$ radius}

The final step was to bend the pixel assembly to a $2 \mathrm{~m}$ radius. This was achieved by gluing the assembly to a concave glass lens held in place using a convex lens pressed with a hydraulic press using $\sim 1000 \mathrm{~kg}$ of force (Fig. 4, step 8). To avoid trapping air bubbles between the bottom of the silicon wafer and the concave lens, the concave side of the lens was diced using a $300 \mu \mathrm{m}$ blade, $2 \mathrm{~mm}$ deep, from the edge of the lens, on a $20 \mathrm{~mm} \times 20 \mathrm{~mm}$ grid. A small drop $(5-10 \mu \mathrm{L})$ of EPO-TEK 301-2FL glue was placed at the center of each die of the concave lens, and then the quartz/silicon wafer was placed between the concave and convex lens and kept under pressure for four days to allow the glue to cure at room temperature. All fabrication steps are summarized by the schematic in Fig. 4.

\section{Tests and results}

The RIXS measurements were performed at beamline 27-ID at the APS. An incident beam of $11.215 \mathrm{keV}$ was generated by two $30 \mathrm{~mm}$-period undulators with a total length of $4.8 \mathrm{~m}$ and monochromated using a water-cooled double-crystal diamond

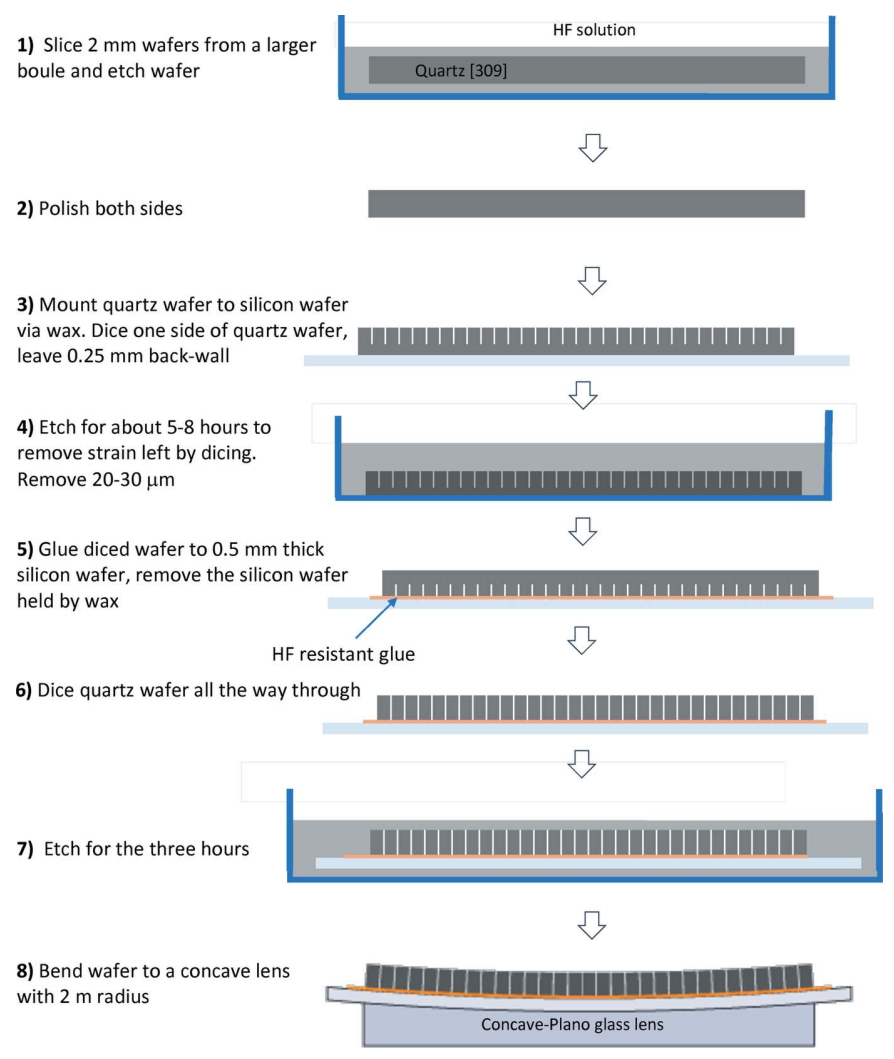

Figure 4

Schematic showing the fabrication steps. high-heat load monochromator. The bandpass of the incident beam was further reduced to $8.9 \mathrm{meV}$ using a four-bounce symmetric Si (844) high-resolution monochromator. Finally, the beam was focused to a spot size of $10 \mu \mathrm{m} \times 40 \mu \mathrm{m}$ FWHM $(\mathrm{V} \times \mathrm{H})$ on the sample using a KB-focusing mirror system. The sample (scattering source), spherical diced analyzer and position-sensitive detector (Mythen by Dectris) were placed on a $2 \mathrm{~m}$-diameter Rowland circle. The position-sensitive detector with $50 \mu \mathrm{m}$ pitch was used in a dispersive geometry (Huotari et al., 2005; Shvyd'ko et al., 2013).

\subsection{Resolution function}

For the spectral resolution function measurement, elastically scattered radiation from a $3 \mathrm{M}$ Scotch tape sample was used and the RIXS spectrometer arm was positioned at a $2 \theta$ scattering angle of $15^{\circ}$. Fig. 5( $\left.a\right)$ shows the measured elastic scattering (spectral resolution function). The $x$-axis is the energy loss, which is defined as the difference between the incident energy and the scattered energy; positive (negative) means an energy loss (gain). The green line is a Voigt function fit with a FWHM of $10.53 \pm 0.1 \mathrm{meV}$. This value is consistent with the approximated FWHM of $10.5 \mathrm{meV}$, resulting from the quadrature of contributions,

$$
\Delta E_{\text {total }}=\left[\Delta E_{\text {mono }}^{2}+\Delta E_{\text {ana }}^{2}+\Delta E_{\text {geom }}^{2}\right]^{1 / 2},
$$

where $\Delta E_{\text {mono }}$ is the incident bandpass $(8.9 \mathrm{meV}), \Delta E_{\text {ana }}$ is the intrinsic analyzer Darwin width contribution $(3.7 \mathrm{meV})$ and $\Delta E_{\text {geom }}$ is the geometric detector and spot-size contributions $(4.2 \mathrm{meV})$. In the energy-gain side, the measured spectral function agrees reasonably with the fitted spectral function; however, in the energy-loss side, a small shoulder peak is seen
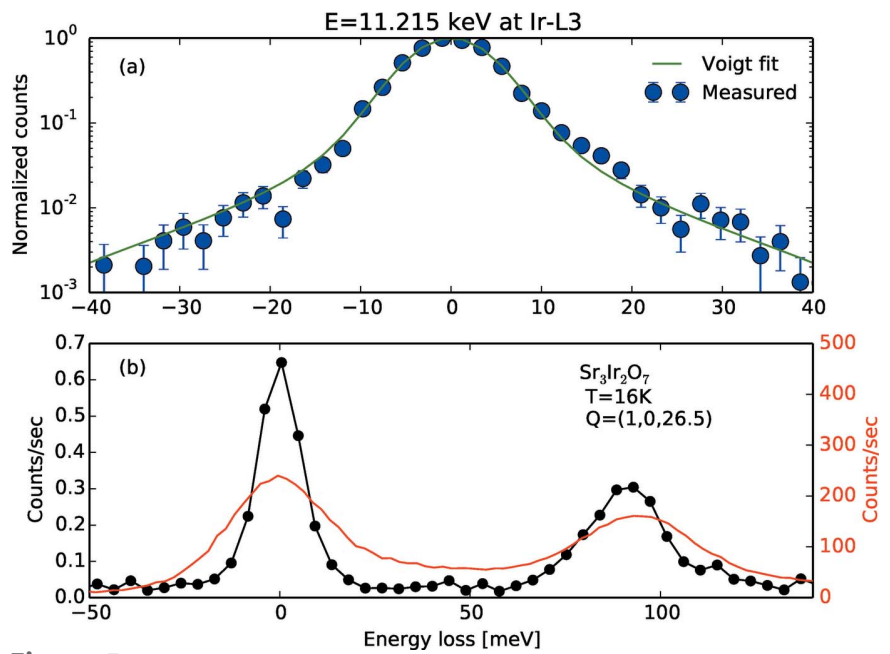

Figure 5

Spectral resolution function and magnon spectrum in $\mathrm{Sr}_{3} \mathrm{Ir}_{2} \mathrm{O}_{7}$. (a) The measured resolution function of the quartz (309) diced spherical analyzer (blue filled circles) and a fitted resolution function (green line), FWHM of $10.53 \pm 0.1 \mathrm{meV}$. (b) Magnon spectrum in $\mathrm{Sr}_{3} \mathrm{Ir}_{2} \mathrm{O}_{7}$ at momentum transfer $Q=(1,0,26.5)$ measured with with quartz analyzer $(10.53 \mathrm{meV}$ : black line, left axis) and $\mathrm{Si}(844)$ analyzer ( $30 \mathrm{meV}$ : red line, right axis). The peak at the zero energy loss is the elastic scattering and the magnon peak at $\sim 90 \mathrm{meV}$. 
at around $17 \mathrm{meV}$ in the measured data. This small shoulder peak probably originates from residual strain in the quartz die.

\subsection{Spectral resolution function and magnon spectrum in} $\mathrm{Sr}_{3} \mathrm{Ir}_{2} \mathrm{O}_{7}$ at the magnetic zone center

A previous RIXS study with $30 \mathrm{meV}$ energy resolution revealed an exceptionally large magnon gap of $\sim 90 \mathrm{meV}$, shown by the red curve in Fig. 5(b), which arises from bonddirectional pseudodipolar interactions that are strongly enhanced near the metal-insulator transition boundary in the spin-orbit-coupled iridates (Kim et al., 2012). Its well defined magnon peak at high energy loss is a suitable feature to check whether the new high-resolution analyzer correctly performs over a wider energy range. Note that this spin-wave feature has a natural width much smaller than the expected instrument resolution and therefore the resulting spectra will be resolution limited. Fig. 5(b) shows the magnon spectrum in $\mathrm{Sr}_{3} \mathrm{Ir}_{2} \mathrm{O}_{7}$ at the momentum transfer $Q=(1,0,26.5)$ as filled black circles. The peak at zero energy loss is the elastic scattering. A well defined magnon peak is clearly resolved at the expected energy-loss value. The acquisition time for the data taken with the quartz analyzer [black curve in Fig. 5(b)] was about $6 \mathrm{~h}$, while the acquisition time for the data taken with the $\mathrm{Si}(844)$ analyzer [red curve in Fig. 5(b)] was about $30 \mathrm{~min}$. The measurements with the quartz analyzer were performed using a small circular mask ( $\sim 18 \mathrm{~mm}$ diameter), in contrast to the $\mathrm{Si}$ analyzer, which was unmasked (100 $\mathrm{mm}$ diameter).

\section{Conclusion}

In this work the fabrication steps for manufacturing a quartz (309) diced spherical analyzer have been reported. The overall energy resolution achieved is $10.53 \mathrm{meV}$, consistent with an expected value of $10.5 \mathrm{meV}$. While the prototype quartz analyzer described in this work achieved the expected energy resolution, more research and development efforts are still needed to further improve the fabrication process to produce larger area analyzers.

\section{Acknowledgements}

The submitted manuscript has been created by UChicago Argonne, LLC, Operator of Argonne National Laboratory. The US Government retains for itself, and others acting on its behalf, a paid-up non-exclusive, irrevocable worldwide license in said article to reproduce, prepare derivative works, distribute copies to the public, and perform publicly and display publicly, by or on behalf of the Government. The Department of Energy will provide public access to these results of federally sponsored research in accordance with the DOE Public Access Plan. (http://energy.gov/downloads/doe-public access-plan). Use of the Center for Nanoscale Materials, an Office of Science user facility, was supported by the US Department of Energy, Office of Science, Office of Basic Energy Sciences, under Contract No. DE-AC02-11357.

\section{Funding information}

Funding for this research was provided by: US Department of Energy Office of Science laboratory, operated under Contract No. DE-AC02-06CH11357.

\section{References}

Ament, L. J. P., van Veenendaal, M., Devereaux, T. P., Hill, J. P. \& van den Brink, J. (2011). Rev. Mod. Phys. 83, 705-767.

Bloss, F. D. \& Gibbs, G. V. (1963). Am. Mineral. 48, 821.

Gog, T., Casa, D. M., Said, A. H., Upton, M. H., Kim, J., Kuzmenko, I., Huang, X. \& Khachatryan, R. (2013). J. Synchrotron Rad. 20, 74-79.

Honnicke, M. G., Bianco, L. M., Ceppi, S. A., Cusatis, C., Huang, X., Cai, Y. Q. \& Stutz, G. E. (2016). J. Appl. Cryst. 49, 1443-1453.

Hönnicke, M. G., Huang, X., Cusatis, C., Koditwuakku, C. N. \& Cai, Y. Q. (2013). J. Appl. Cryst. 46, 939-944.

Huotari, S., Vankó, Gy., Albergamo, F., Ponchut, C., Graafsma, H., Henriquet, C., Verbeni, R. \& Monaco, G. (2005). J. Synchrotron Rad. 12, 467-472.

Ketenoglu, D., Harder, M., Klementiev, K., Upton, M., Taherkhani, M., Spiwek, M., Dill, F.-U., Wille, H.-C. \& Yavaş, H. (2015). J. Synchrotron Rad. 22, 961-967.

Kim, J., Said, A. H., Casa, D., Upton, M. H., Gog, T., Daghofer, M., Jackeli, G., van den Brink, J., Khaliullin, G. \& Kim, B. J. (2012). Phys. Rev. Lett. 109, 157402.

Kosinski, J. A., Gualtieri, J. G. \& Ballato, A. (1991). Proceedings of the 45th Annual Symposium on Frequency Control, 29-31 May 1991, Los Angeles, CA, USA, pp. 22-28.

Kushnir, V. I. \& Suvorov, E. V. (1990). Phys. Status Solidi. (A), 122, 391-404.

Masciovecchio, C., Bergmann, U., Krisch, M., Ruocco, G., Sette, F. \& Verbeni, R. (1996). Nucl. Instrum. Methods Phys. Res. B, 111, 181186.

Schwoerer-Böehning, M., Abbamonte, P. M., Macrander, A. T. \& Kushnir, V. I. (1997). Proc. SPIE, 3151, 282-286.

Sette, F., Ruocco, G., Krisch, M., Bergmann, U., Masciovecchio, C., Mazzacurati, V., Signorelli, G. \& Verbeni, R. (1995). Phys. Rev. Lett. 75, 850-853.

Shvyd'ko, Y. V. (2004). X-ray Optics - High-Energy-Resolution Applications, Vol. 98 of Springer Series in Optical Sciences. Heidelberg: Springer.

Shvyd'ko, Y. V., Hill, J., Burns, C., Coburn, D., Brajuskovic, B., Casa, D., Goetze, K., Gog, T., Khachatryan, R., Kim, J.-H., Kodituwakku, C., Ramanathan, M., Roberts, T., Said, A., Sinn, H., Shu, D., Stoupin, S., Upton, M., Wieczorek, M. \& Yavas, H. (2013). J. Electron Spectrosc. Relat. Phenom. 188, 140-149.

Sutter, J. P., Baron, A. Q., Ishikawa, T. \& Yamazaki, H. (2005). J. Phys. Chem. Solids, 66, 2306-2309.

Sutter, J. P., Baron, A. Q. R., Miwa, D., Nishino, Y., Tamasaku, K. \& Ishikawa, T. (2006). J. Synchrotron Rad. 13, 278-280.

Yavaş, H., Ercan Alp, E., Sinn, H., Alatas, A., Said, A. H., Shvyd'ko, Y., Toellner, T., Khachatryan, R., Billinge, S. J., Zahid Hasan, M. \& Sturhahn, W. (2007). Nucl. Instrum. Methods Phys. Res. A, 582, 149-151. 